\title{
Efecto del zinc aminoquelado y el sulfato de zinc en la incidencia de la infección respiratoria y la diarrea en niños preescolares de centros infantiles
}

\author{
Juliana Sánchez¹, Oscar Alonso Villada1, Maylen Liseth Rojas ${ }^{1}$, Liliana Montoya ${ }^{1,2}$, Alejandro Díaz,3, \\ Cristian Vargas $^{1,4}$, Javier Chica ${ }^{1,5}$, Ana Milena Herrera ${ }^{1}$ \\ 1 Grupo de Investigación Ciencias Básicas, Facultad de Medicina, Universidad CES, Medellín, Colombia \\ 2 Dirección de Investigación, Facultad de Medicina, Universidad CES, Medellín, Colombia \\ ${ }^{3}$ Cátedra de Pediatría, Facultad de Medicina, Universidad CES, Medellín, Colombia. \\ ${ }^{4}$ Maestría en Epidemiologia, Facultad de Medicina, Universidad CES, Medellín, Colombia \\ ${ }^{5}$ Centro de Investigación y Medición, PREMEX, Medellín, Colombia
}

Introducción. El déficit de zinc es común en niños de poblaciones en desarrollo. La deficiencia de zinc altera el sistema inmunológico y la resistencia a las infecciones.

Objetivo. Evaluar el efecto de dos compuestos de zinc en la prevención de la infección respiratoria y la diarrea agudas.

Materiales y métodos. Se llevó a cabo un ensayo comunitario triple ciego en 301 niños entre dos y cinco años de edad de centros infantiles de Medellín. Fueron seis conglomerados distribuidos aleatoriamente en tres grupos de intervención que recibieron zinc aminoquelado, sulfato de zinc y placebo durante cinco días de la semana a lo largo de 16 semanas. Se evaluaron diariamente los síntomas de infección respiratoria, diarrea aguda y efectos secundarios.

Resultados. La incidencia de la infección respiratoria fue menor con el zinc aminoquelado (1,42 por 1.000 días-niño) comparado con el sulfato de zinc (1,57 por 1.000 días-niño) ( $R R=0,90, I_{95 \%}: 0,382$ a $2,153, p=0,999)$ y con el placebo (3,3 por 1.000 días-niño) $\left(R R=0,43, I C_{95 \%}: 0,196\right.$ a 0,950, $\left.p=0,049\right)$. La incidencia de diarrea aguda con el zinc aminoquelado $(0,15$ por 1.000 días-niño) fue menor que con el sulfato de zinc (1,18 0,78 por 1.000 días-niño) $\left(R R=0,20, I_{95 \%}: 0,0043\right.$ a $\left.1,662, p=0,361\right)$ y que la del grupo placebo ( 0,49 por 1.000 días-niño) ( $R R=0,32, I_{95 \%}: 0,006$ a 3,990, $\left.p=0,346\right)$.

Conclusiones. El zinc aminoquelado tuvo mejor efecto en la disminución de la incidencia de la infección respiratoria y la diarrea agudas en niños preescolares comparado con los otros grupos de estudio.

Palabras clave: infecciones del sistema respiratorio/epidemiología, diarrea, zinc, placebos.

Identificador en clinicaltrials.gov: NCT01791608

doi: http://dx.doi.org/10.7705/biomedica.v34i1.1581

Effect of zinc amino acid chelate and zinc sulfate in the incidence of respiratory infection and diarrhea among preschool children in child daycare centers

Introduction: Zinc deficiency is common in children among populations in developing areas. Zinc deficiency alters the immune system and the resistance to infections.

Objective: To evaluate the effect of two zinc compounds in the prevention of acute respiratory infection and acute diarrhea.

Materials and methods: Randomized triple-blind community trial with 301 children between 2-5 years of age from six child daycare centers in Medellin, Colombia. Children were distributed in three groups receiving zinc amino acid chelate, zinc sulfate and placebo five days a week for 16 weeks. Daily symptoms of respiratory infection, acute diarrhea and side effects were evaluated.

Results: The incidence of respiratory infection was lower with zinc amino acid chelate $(1.42$ per 1,000 child-days) compared with placebo (3.3 per 1,000 child-days) ( $\mathrm{RR}=0.43,95 \% \mathrm{Cl}: 0.196$ to 0.950 , $\mathrm{p}=0.049)$ and with zinc sulfate ( 1.57 per 1,000 child-days) $(R R=0.90,95 \% \mathrm{Cl} 0.382$ to $2.153, p=0.999)$. The incidence of acute diarrhea with zinc amino acid chelate ( 0.15 per 1,000 child-days) was lower than with placebo ( 0.49 per 1,000 child-days $)(R R=0.32,95 \% \mathrm{Cl} 0.006$ to $3.990, p=0.346)$ and with zinc sulfate ( 0.78 per 1,000 child-days) $(R R=0.20,95 \% \mathrm{Cl}: 0.0043$ to $1.662, p=0.361)$.

\section{Contribución de los autores:}

Juliana Sánchez y Oscar Villada: análisis estadístico, interpretación de los resultados y redacción del manuscrito.

Liliana Patricia Montoya, Ana Milena Herrera, Maylen Liseth Rojas, Alejandro Díaz, Javier Chica y Cristian Vargas: análisis de los resultados.

Todos los autores participaron en el diseño y ejecución del estudio, la revisión y la aprobación de la versión final del manuscrito 
Conclusions: Zinc amino acid chelate had a better effect in reducing the incidence of acute respiratory infection and acute diarrhea in preschool children when compared with the other groups.

Keywords: Respiratory tract infections/epidemiology, diarrhea, zinc, placebos. Identifier in clinicaltrials.gov: NCT01791608 doi: http://dx.doi.org/10.7705/biomedica.v34i1.1581

El zinc es un micronutriente esencial en el crecimiento celular, la síntesis proteica y la diferenciación celular. Como consecuencia, la deficiencia de zinc afecta el crecimiento físico de los niños y aumenta el riesgo y la gravedad de una variedad de infecciones $(1,2)$.

Más de $60 \%$ de los niños menores de cinco años tienen carencia de zinc debido a esquemas alimentarios inadecuados en algunos países de bajos ingresos y en desarrollo (3).

Según el International Zinc Nutrition Consultative Group (IZiNCG), Colombia se clasifica en una categoría de riesgo moderado de deficiencia de zinc (4). Los datos de la Encuesta Nacional de la Situación Nutricional en Colombia (ENSIN) del 2010 muestran que $43 \%$ de los niños entre 1 y 4 años tienen deficiencia de zinc (5).

Diversos estudios sugieren que las poblaciones con déficit de zinc presentan mayor riesgo de desarrollar enfermedades diarreicas, infecciones de las vías respiratorias y retraso del crecimiento. Se estima que la deficiencia de zinc está asociada con cerca de 800.000 muertes anuales a nivel mundial entre niños menores de cinco años, incluidas las muertes atribuibles a diarrea (176.000) y neumonía (406.000) (1).

Según estimaciones de la Organización Mundial de la Salud (OMS) y de la UNICEF, en el año 2008 ocurrieron 8.795 millones de muertes en niños menores de cinco años, $68 \%$ de las cuales se debió a enfermedades infecciosas, $18 \%$, a la neumonía y $15 \%$, a la diarrea (6).

Según los datos de la Dirección Seccional de Salud de Antioquia, en este departamento la primera causa de morbilidad en consulta externa y urgencias en niños menores de cinco años durante el 2010 fue el resfriado común, seguido de la diarrea aguda, mientras que la infección

\footnotetext{
Correspondencia:

Juliana Sánchez, Carrera 81 B № 7A-40, interior 120, Medellín, Colombia

Teléfonos: (574) 347 3771, 2319331 y (300) 7771707

july912@yahoo.com.ar

Recibido: 04/04/13; aceptado: 22/08/13
}

aguda de las vías respiratorias superiores se ubicó como la sexta causa más común de morbilidad en ese grupo de edad (7).

El uso de zinc como una terapia adjunta en el tratamiento de la diarrea aguda mejora la eficacia del manejo y se convierte en una acción costoefectiva comparada con el manejo estándar de la diarrea $(8,9)$.De igual forma, varios metaanálisis han demostrado que podría disminuir la incidencia de la diarrea aguda cuando se toma por varias semanas (10-12); no obstante, su uso en la prevención no está aprobado. Respecto a la infección respiratoria aguda, varios metaanálisis han mostrado resultados contradictorios tanto en el tratamiento como en la prevención de la neumonía y del resfriado común con suplementos de zinc $(10,12-14)$.

En nutrición infantil se utilizan varios tipos de compuestos de zinc (4), siendo el sulfato de zinc uno de los más usados. A pesar de su seguridad y baja toxicidad, estos compuestos pueden desencadenar efectos secundarios como náuseas, vómitos y dolor abdominal (15-17), sin mencionar su sabor desagradable, por lo que es necesario buscar vehículos que enmascaren su sabor para administrarlo a niños.

Existen escasos estudios con compuestos de zinc aminoquelado, los cuales muestran mayor absorción intestinal, mayor incremento en los niveles de zinc en sangre y mejor tolerancia digestiva $(17,18)$; no se encontró información que estableciera cuál compuesto es más efectivo en niños preescolares.

Si hubiera mayor información científica sobre el compuesto de zinc más efectivo, mejor tolerado y en presentaciones con vehículos de fácil consumo, se podría ayudar a los productores de complementos y alimentos fortificados con zinc a tomar decisiones a la hora de seleccionar el compuesto que se utilice, de forma que exista buena observancia en su consumo por parte de la población infantil y se pueda contribuir así a disminuir la alta carga de morbimortalidad infantil por infecciones respiratorias y enfermedad diarreica aguda. 
Se realizó un ensayo comunitario con el objetivo de evaluar el efecto de dos compuestos de zinc en la prevención de la infección respiratoria y la enfermedad diarreica agudas en niños preescolares de seis centros infantiles de Medellín.

\section{Materiales y métodos}

\section{Participantes}

Se llevó a cabo un ensayo comunitario con muestreo por conglomerados no probabilístico; se seleccionaron seis centros infantiles de la fundación FAN de Medellín, cada centro infantil se consideró como un conglomerado, asignándose dos centros a cada grupo de intervención para así poder disminuir la correlación intraclase. La intervención se realizó entre junio y septiembre de 2012. Todos los niños de cada conglomerado fueron invitados a participar en la investigación y participaron en el estudio los que cumplieron los criterios de inclusión y exclusión.

\section{Criterios de inclusión}

Se incluyeron niños de entre dos y cinco años de edad al inicio del estudio que asistieran ocho horas diarias al centro infantil, recibieran más del $80 \%$ de su alimentación diaria en la institución y les gustara la leche.

\section{Criterios de exclusión}

Se excluyeron los niños con diagnóstico médico de neumonía recurrente, fibrosis quística, malformaciones digestivas, diarrea persistente por cualquier causa, enfermedad inflamatoria intestinal o intolerancia a la lactosa, y los niños que en el momento de inicio de la intervención presentaban infección respiratoria o enfermedad diarreica aguda diagnosticada por médico, así como los niños que hubieran recibido complementos de zinc en los dos meses previos al inicio del estudio, aunque no los estuvieran recibiendo en el momento del inicio de la intervención.

El tamaño de la muestra se determinó por conveniencia, se calculó nuevamente el poder con los 301 niños participantes en el estudio teniendo en cuenta las siguientes diferencias en las proporciones establecidas según la literatura: para la disminución en la incidencia de la infección respiratoria aguda con el consumo de zinc, 19 $\%$, y para la disminución de la incidencia de la enfermedad diarreica aguda, $14,9 \%$, resultando para la primera un poder de $87,4 \%$ y para la segunda de $67,8 \%$, con un intervalo de confianza de $95 \%$ para ambas.

\section{Asignación aleatoria y enmascaramiento}

Se asignaron en forma aleatoria, por medio de balota, los seis conglomerados de manera que dos centros infantiles quedaron en cada uno de los tres grupos de estudio. Un grupo recibió sulfato de zinc, otro grupo recibió zinc aminoquelado y el último grupo recibió placebo.

La dosis de zinc fue de $7 \mathrm{mg}$ en niños de dos a tres años y de 9,45 mg en niños de cuatro a cinco años (dosis recomendadas por el IZINCG). El vehículo utilizado fue leche fortificada con el respectivo compuesto de zinc; los tres suplementos lácteos fueron similares en color, olor y sabor. El laboratorio NUTREO (Rionegro, Colombia) se encargó de suministrarlos. El estudio fue triple ciego durante toda la intervención y análisis de la información.

\section{Intervención}

Se aplicó un formulario estandarizado por parte de personal entrenado para recolectar los datos de las variables sociodemográficas. Los profesionales que obtuvieron los datos de las encuestas realizaron las preguntas en cada centro infantil a la misma hora, con el mismo tono de voz y buscando no inducir las respuestas. Nutricionistas entrenadas tomaron las medidas antropométricas (peso y talla) con pesas y tallímetros calibrados antes de iniciar la intervención y a las 16 semanas cuando finalizó.

El personal del restaurante escolar suministró la leche (complementada o no, según el caso) a los preescolares después de recibir las instrucciones y de ser entrenado por los investigadores. La dosis diaria se suministró en dos tomas, una en el desayuno y otra en la merienda, de lunes a viernes durante 16 semanas. Las docentes de cada salón fueron capacitadas y entrenadas para registrar -con ayuda de los investigadores- los datos diarios de asistencia, consumo de la bebida láctea y síntomas de infección respiratoria o diarrea, y los efectos secundarios de los complementos con zinc y sin él (dolor abdominal, vómito y sabor desagradable) referidos por el niño y el cuidador. El registro se hizo diariamente de lunes a viernes con el fin de disminuir el sesgo de memoria.

\section{Definiciones}

Se definió como infección respiratoria aguda la presencia de dos o más de los siguientes síntomas: tos, rinorrea, dificultad para respirar y dolor faríngeo de dos o más días de evolución. Como caso nuevo se consideró el transcurso entre dos episodios de cinco o más días sin dos o más de los síntomas previamente descritos. 
La enfermedad diarreica aguda se definió como la ocurrencia de tres o más deposiciones líquidas en 24 horas con más de dos días de evolución y menos de 14 días de duración. Como caso nuevo se consideró el transcurso de tres o más días libres de diarrea entre dos episodios.

Se definió como vómito y dolor abdominal por ingestión del complemento un episodio ocurrido en los primeros veinte minutos después de la ingestión de la bebida láctea enriquecida.

\section{Estandarización}

El personal encargado de realizar las encuestas, el personal que preparó y suministró la leche en los centros infantiles y los encargados de registrar los datos de morbilidad fue instruido y entrenado previamente. Cada 15 días se verificó el adecuado suministro y toma de los suplementos por parte de los niños, así como el adecuado diligenciamiento de los registros.

Para la toma del peso en los niños se utilizó una balanza electrónica marca Seca $803^{\circledR}$ con una precisión de $100 \mathrm{~g}$ y capacidad de $150 \mathrm{~kg}$. Para la medición se indicó a los niños que se retiraran los zapatos y se procuró que estuvieran con la menor cantidad de ropa posible. Para la toma de la talla se utilizaron tallímetros de marca Seca $213^{\circledR}$ con precisión de $1 \mathrm{~mm}$ y capacidad de dos metros. Se realizó la medición sin zapatos y en las niñas sin moños ni peinados que pudieran interferir con la medida.

Se hizo una prueba piloto con 25 padres para la valoración de los instrumentos de consentimiento informado y el formulario de reclutamiento; además, en 25 niños se realizaron pruebas de aceptación del producto lácteo con zinc o sin él y del enmascaramiento de las leches fortificadas, de forma que se parecieran lo más posible en sabor y presentación. En la prueba piloto se evaluó también el formulario sobre morbilidad y efectos secundarios, así como la comprensión y adecuada toma de los datos por parte de las docentes encargadas.

El laboratorio produjo las leches fortificadas cada mes y se encargó de entregarlas al centro infantil correspondiente también mensualmente manteniendo el enmascaramiento.

\section{Consideraciones éticas}

La investigación fue aprobada por el Comité de Ética de la Universidad CES, el cual clasificó el estudio con un riesgo mayor que el mínimo; se siguieron las normas éticas del comité institucional y de la Declaración de Helsinki, así como las consideraciones de la Resolución 8430 de 1993 del Ministerio de Salud de Colombia.

\section{Estadística}

En el análisis estadístico, primero se resumió la información de las variables continuas utilizando promedios con su correspondiente desviación estándar, y las variables categóricas, con proporciones; segundo, para la determinación de las diferencias estadísticas entre la variable respuesta y las covariables, las variables cuantitativas se compararon en los tres grupos por medio de la prueba no paramétrica de Kruskal-Wallis, ya que no siguieron una distribución normal. Para medir la asociación de las variables categóricas se usó la prueba de ji al cuadrado de independencia o, en su defecto, la prueba exacta de Fisher, dependiendo del número esperado de frecuencias menores que cinco. En todos los casos, el nivel de significación utilizado fue de $5 \%$. Se valoró el estado nutricional antes y después de la intervención utilizando las aplicaciones gratuitas de la Organización Mundial de la Salud: la WHO Anthro, versión 3.2.2 de 2011, en el caso de los menores de cinco años, y la WHO Anthro plus, versión 1.0.4, para los niños con cinco años y más.

Se calcularon y se compararon las incidencias de IRA y enfermedad diarreica aguda en los tres grupos de estudio, estimando el riesgo relativo, y su respectivo intervalo de confianza de $95 \%$, así como el valor de p. Se calculó el porcentaje de reducción del riesgo relativo $(\mathrm{RR})$ y el número necesario de tratar cuando fue pertinente.

Se analizó si la variable alergia se comportaba como posible elemento de confusión en el análisis de la infección respiratoria, pues los niños que presentaban algún tipo de alergia no alteraban el cálculo de la incidencia de la enfermedad diarreica aguda, pero sí podían afectar el análisis de la incidencia de la infección respiratoria aguda.

En el análisis de tiempo-persona, la tasa de incidencia de la enfermedad diarreica y de la infección respiratoria aguda fue el número de episodios nuevos sobre el número de días-niño por observación.

El periodo de observación de cada niño se contó a partir del día de inicio del suministro de los compuestos con zinc o sin él hasta la semana 16, cuando terminó la intervención.

Se calculó la proporción de efectos secundarios en cada grupo y se compararon con la prueba de ji al cuadrado de independencia, calculando 
su respectivo valor de $\mathrm{p}$ (el nivel de significación utilizado fue de $5 \%$ ).

La información se digitalizó en una hoja de cálculo de MS Office Excel $2007^{\text {TM }}$ y luego se exportó, almacenó y procesó en una base de datos en el programa estadístico SPSS ${ }^{\text {TM }}$, versión 21; para el cálculo de los intervalos de confianza y algunas pruebas de asociación se utilizó la calculadora epidemiológica Epidat, versión 3.0, en tanto que la presentación tabular, pictórica y textual se realizó mediante el procesador de texto de MS Office Word 2007 ${ }^{\mathrm{TM}}$.

\section{Resultados}

Se invitaron a participar a 441 niños, es decir, el total de preescolares entre dos y cinco años de edad que asistía a seis centros infantiles de la Fundación de
Atención de la Niñez (FAN) de Medellín. De estos, 67 niños no cumplían los criterios de inclusión y 17 se retiraron de los centros infantiles antes de iniciar la intervención por motivos de orden público en algunas comunas de la ciudad. Quedaron, entonces, 357 menores que recibieron los complementos con zinc o sin él durante 16 semanas (entre el 4 de junio y el 22 de septiembre del 2012); de estos, 17 $(4,76 \%)$ se perdieron durante el seguimiento de la intervención y 39 fueron excluidos del análisis final por haber ingerido la leche durante menos de $75 \%$ del tiempo de observación definido por el protocolo. Así, el análisis se hizo con 301 infantes: 93 de ellos tomaron leche fortificada con zinc aminoquelado, 112, leche fortificada con sulfato de zinc y 96 recibieron placebo (figura 1).

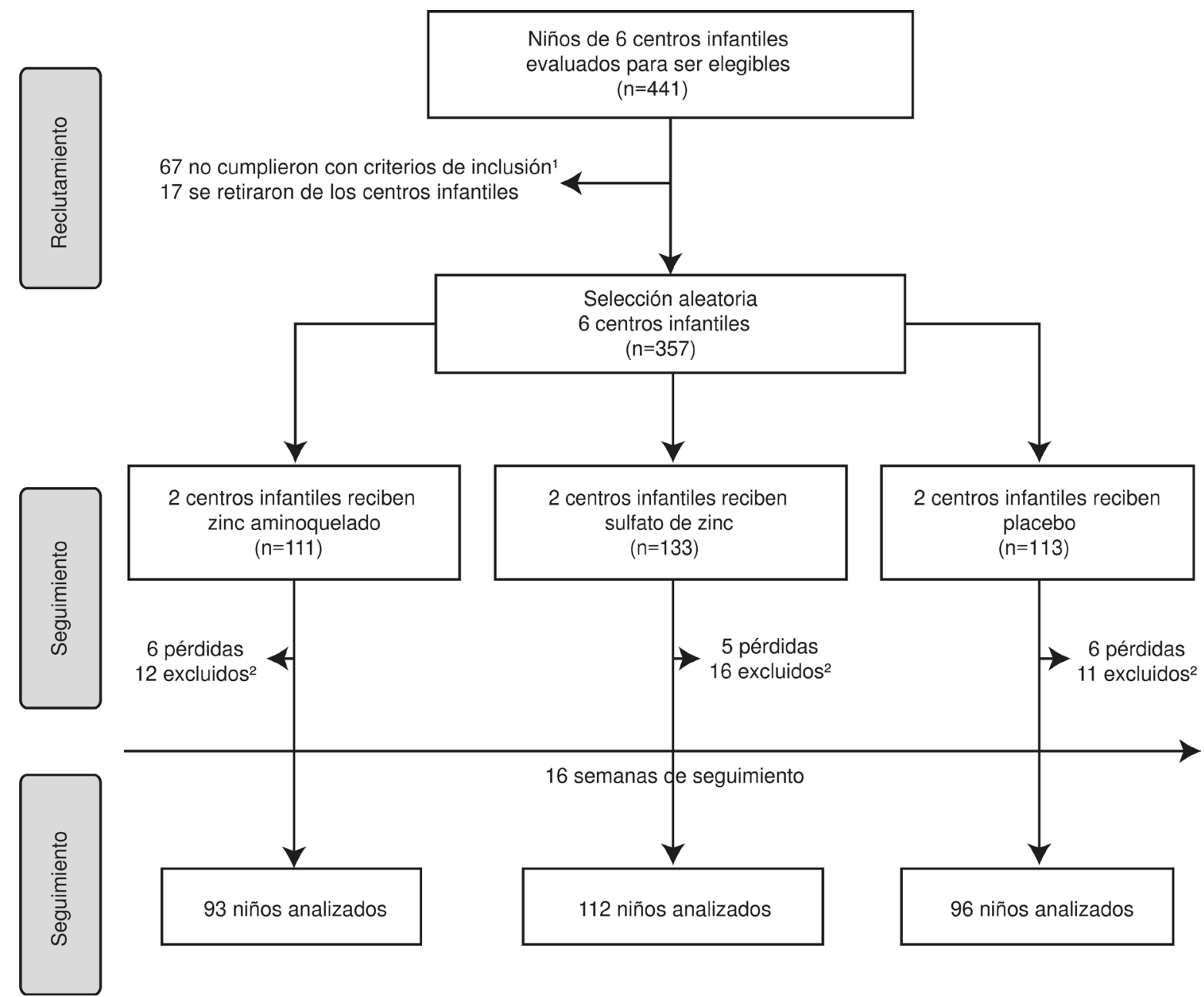

${ }^{1}$ No cumplían con la edad ni presentaban diarrea persistente, intolerancia a la lactosa, o no les gustaba la leche.

${ }^{2}$ Niños que recibieron los complementos con zinc y sin él durante $75 \%$ o menos de los días de consumo ( $<55$ días), o niños que dejaron de asistir a los centros infantiles 15 días hábiles consecutivos.

Figura 1. Diagrama de flujo del efecto preventivo del zinc aminoquelado y el sulfato de zinc en la infección respiratoria y la diarrea aguda 
Cuadro 1. Características sociales y demográficas de los niños preescolares según grupo de estudio

\begin{tabular}{|c|c|c|c|c|c|}
\hline & $\begin{array}{l}\text { Zinc aminoquelado } \\
\qquad(n=93)\end{array}$ & $\begin{array}{l}\text { Sulfato de zinc } \\
\qquad(n=112)\end{array}$ & $\begin{array}{c}\text { Placebo } \\
(n=96)\end{array}$ & $\begin{array}{l}\text { Total } \\
(\mathbf{3 0 1})\end{array}$ & $\mathbf{p}^{2}$ \\
\hline & n (\%) & n (\%) & n (\%) & n (\%) & \\
\hline \multicolumn{6}{|l|}{ Sexo $^{1}$} \\
\hline Hombre & $54(58,1)$ & $52(46,4)$ & $51(53,1)$ & $157(52,1)$ & 0,245 \\
\hline Mujer & $39(41,9)$ & $60(53,6)$ & $45(45,9)$ & $144(47,9)$ & \\
\hline \multicolumn{6}{|c|}{ Estrato socioeconómico de la vivienda ${ }^{1}$} \\
\hline Estrato 1 & $13(14)$ & $12(10,7)$ & $25(26)$ & $50(16,6)$ & $0,008 *$ \\
\hline Estrato 2 & $28(30,1)$ & $18(16,1)$ & $50(52,1)$ & $96(31,9)$ & $0,000^{* *}$ \\
\hline Estratos 3-4 & $52(55,9)$ & $82(72,2)$ & $21(21,9)$ & $155(51,5)$ & $0,000 *$ \\
\hline \multicolumn{6}{|c|}{ Tipo de tenencia de la vivienda ${ }^{1}$} \\
\hline Propia & $19(20,4)$ & $42(37,5)$ & $29(30,2)$ & $90(29,9)$ & $0,029^{* *}$ \\
\hline Arrendada & $66(71)$ & $63(56,3)$ & $63(65,6)$ & $192(63,8)$ & 0,083 \\
\hline Otra & $8(8,6)$ & $7(6,3)$ & $4(4,2)$ & $19(6,3)$ & 0,455 \\
\hline \multicolumn{6}{|l|}{ Tipo de familia ${ }^{1}$} \\
\hline Nuclear & $42(45,2)$ & $40(35,7)$ & $41(42,7)$ & $123(40,9)$ & 0,354 \\
\hline Extensa o conjunta & $19(20,4)$ & $13(11,6)$ & $7(7,3)$ & $39(12,9)$ & $0,023^{* *}$ \\
\hline Monoparental & $32(34,4)$ & $59(52,7)$ & $48(50)$ & $139(46,2)$ & $0,021^{* *}$ \\
\hline \multicolumn{6}{|c|}{ Tipo de afiliación del niño al SGSS ${ }^{1}$} \\
\hline Subsidiado & $59(63,4)$ & $72(64,3)$ & $55(57,3)$ & $186(61,8)$ & 0,541 \\
\hline Contributivo & $34(36,6)$ & $40(35,7)$ & $41(42,7)$ & $115(38,2)$ & \\
\hline \multicolumn{6}{|l|}{ Población desplazada ${ }^{1}$} \\
\hline Sí & $11(11,8)$ & $13(11,6)$ & $15(15,6)$ & $39(13)$ & 0,640 \\
\hline No & $82(88,2)$ & $99(88,4)$ & $81(84,4)$ & $262(87)$ & \\
\hline \multicolumn{6}{|l|}{ Alérgicos $^{1}$} \\
\hline Sí & $16(17,2)$ & $33(29,5)$ & $23(24)$ & $72(23,9)$ & 0,122 \\
\hline No & $77(82,8)$ & $79(70,5)$ & $73(76)$ & $229(76,1)$ & \\
\hline
\end{tabular}

${ }^{1} \chi^{2}$ de independencia

${ }^{2}$ Significación estadística: $p<0,05$

\section{Caracterización social, demográfica y nutricional}

El $52,1 \%$ de los niños era de sexo masculino, el $50 \%$ tenía tres años de edad o menos al inicio del estudio (rango intercuartílico $(R q)=1$ año). No se encontraron diferencias estadísticas entre los grupos con respecto a la edad (prueba de Kruskal Wallis, $p=0,057$ ) ni el sexo (prueba de $\chi^{2}$ de independencia, $p=0,245$ ). Los grupos fueron comparables respecto a las variables sociodemográficas, a excepción de la de estrato socioeconómico, la tipología familiar y el tipo de tenencia de la vivienda (prueba de $\chi^{2}$ de independencia, $p<0,05$ ) (cuadros 1 y 2). Las variables de estrato, tipología de familia y tenencia de vivienda se controlaron durante el análisis y no se encontró que actuaran como posibles variables de confusión al comparar el RR crudo con el RR ajustado.

Antes de comenzar la intervención, $50 \%$ de los niños tenía un peso de $15,6 \mathrm{~kg}$ o menos $(\mathrm{Rq}=3,03 \mathrm{~kg})$ y una talla de $99,0 \mathrm{~cm}$ o menos $(\mathrm{Rq}=9,4 \mathrm{~cm})$; cuando se comparó la clasificación nutricional de los niños al comienzo del estudio y los indicadores antropométricos de talla para la edad, peso para la edad, peso para la talla e
Cuadro 2. Medidas de tendencia central y dispersión de la variable edad de los niños preescolares según grupo de estudio

\begin{tabular}{lccccc}
\hline & $\begin{array}{c}\text { Zinc } \\
\text { aminoquelado } \\
(\mathrm{n}=93)\end{array}$ & $\begin{array}{c}\text { Sulfato } \\
\text { de zinc } \\
(\mathrm{n}=112)\end{array}$ & $\begin{array}{c}\text { Placebo' } \\
(\mathrm{n}=96)\end{array}$ & $\begin{array}{c}\text { Total }^{2} \\
(\mathrm{n}=301)\end{array}$ & $\mathbf{p}^{2}$ \\
\hline Edad $^{1}$ & & & & & \\
$\bar{x} \pm D E$ & $3,28 \pm 0,73$ & $3,45 \pm 0,78$ & $3,19 \pm 0,92$ & $3,31 \pm 0,81$ & \\
$\mathrm{Me}(\mathrm{Rq})$ & $3(1)$ & $4(1)$ & $3(2)$ & $3(1)$ & 0,057 \\
CV (\%) & 22,3 & 22,6 & 28,8 & 24,5 & \\
\hline
\end{tabular}

$\bar{x} \pm D E \mathbf{X}$ media, desviación estándar; Me: mediana; Rq: rango intercuartílico; CV: coeficiente de variación

${ }^{1}$ Kruskal-Wallis

${ }^{2}$ Significación estadística: $p<0,05$

índice de masa corporal (IMC) para la edad con base en el modelo de puntuación z y de acuerdo a los patrones de crecimiento de la OMS (20), no se encontraron diferencias significativas (prueba de $\chi^{2}$ de independencia, $p>0,05$ ) (cuadro 3 ).

Al finalizar la intervención, los niños fueron pesados y medidos de nuevo, encontrándose aumento significativo en el peso y la talla en los tres grupos (Wilcoxon, $p=0,000$ en los tres grupos) (figura 2).

Cuando se compararon los indicadores nutricionales al finalizar el estudio no se encontraron diferencias estadísticas entre los tres grupos 
Cuadro 3. Indicadores nutricionales al inicio del estudio según grupo

\begin{tabular}{|c|c|c|c|c|c|}
\hline \multirow[t]{2}{*}{$\begin{array}{l}\text { Indicador nutricional } \\
\text { (puntuación z) }\end{array}$} & $\begin{array}{l}\text { Zinc aminoquelado } \\
\qquad(n=93)\end{array}$ & $\begin{array}{l}\text { Sulfato de zinc } \\
\qquad(n=112)\end{array}$ & $\begin{array}{l}\text { Placebo } \\
(n=96)\end{array}$ & $\begin{array}{c}\text { Total } \\
(n=301)\end{array}$ & \multirow[t]{2}{*}{$\mathbf{p}$} \\
\hline & n (\%) & n (\%) & n (\%) & n (\%) & \\
\hline \multicolumn{6}{|l|}{ Peso para la talla* } \\
\hline Bajo peso para la talla ( $\leq 2 \mathrm{DE})$ & $2(2,2)$ & 0 & 0 & $2(0,6)$ & - \\
\hline Riesgo de peso bajo para la talla $(\geq-2$ a $<-1 \mathrm{DE}$ & $6(6,5)$ & $3(2,7)$ & $3(3,1)$ & $12(3,9)$ & 0,339 \\
\hline Peso adecuado para la talla ( $\geq-1$ a $\leq 1 \mathrm{DE})$ & $59(63,4)$ & $78(69,6)$ & $76(79,2)$ & $213(70,7)$ & 0,056 \\
\hline Sobrepeso (>1 a $\leq 2 \mathrm{DE})$ & $17(18,3)$ & $23(20,5)$ & $10(10,4)$ & $50(16,6)$ & 0,129 \\
\hline Obesidad (>2 DE) & $9(9,7)$ & $8(7,1)$ & $7(7,3)$ & $24(7,9)$ & 0,765 \\
\hline \multicolumn{6}{|l|}{ IMC para la edad* } \\
\hline$\leq 1 \mathrm{DE}$ & $68(73,2)$ & $79(70,6)$ & $74(77,1)$ & $221(73,4)$ & 0,564 \\
\hline Sobrepeso (>1 a $\leq 2 \mathrm{DE})$ & $14(15,1)$ & $25(22,3)$ & $15(15,6)$ & $54(17,9)$ & 0,310 \\
\hline Obesidad (>2 DE) & $11(11,8)$ & $8(7,1)$ & $7(7,3)$ & $26(8,6)$ & 0,419 \\
\hline \multicolumn{6}{|l|}{ Peso para la edad* } \\
\hline Peso bajo para la edad (<-2 DE) & $3(3,2)$ & 0 & 0 & $3(0,9)$ & - \\
\hline Peso adecuado para la edad ( $\geq-1$ a $\leq 1 \mathrm{DE})$ & $90(96,8)$ & $112(100)$ & $96(100)$ & $298(99)$ & \\
\hline \multicolumn{6}{|l|}{ Talla para la edad* } \\
\hline Talla baja para la edad (<-2 DE) & $4(4,3)$ & $5(4,5)$ & $7(7,3)$ & $16(5,3)$ & 0,578 \\
\hline Riesgo de talla baja ( $\geq-2$ a $<-1 \mathrm{DE})$ & $20(21,5)$ & $30(26,8)$ & $30(31,3)$ & $80(26,6)$ & 0,316 \\
\hline Talla adecuada para la edad ( $\geq-1 \mathrm{DE})$ & $69(74,2)$ & $77(68,8)$ & $59(61,5)$ & $205(68,1)$ & 0,168 \\
\hline
\end{tabular}

${ }^{*} \chi^{2}$ de independencia

** Significación estadística: $p<0,05$
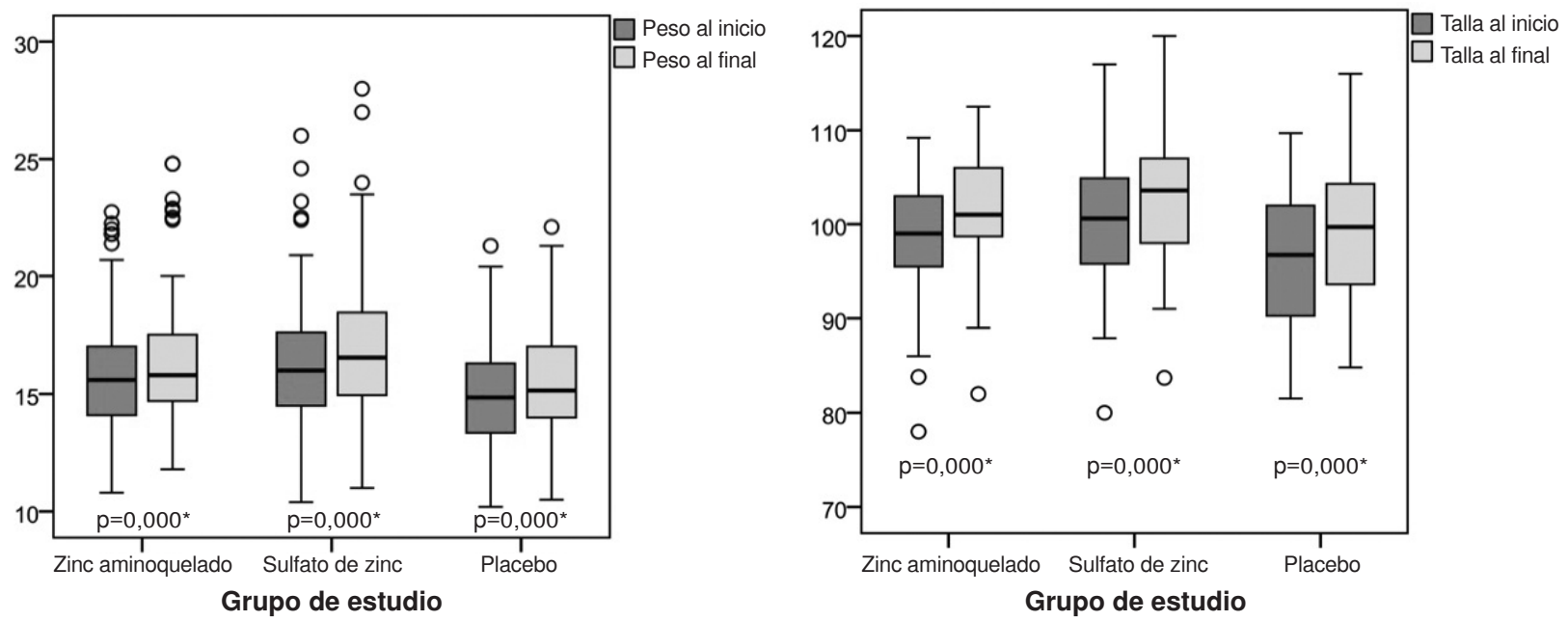

* Wilcoxon

Significación estadística: $p<0,05$

Figura 2. Peso y talla antes y después de la intervención según grupo de estudio

respecto al peso para la edad $(p=0,295)$, peso para la talla $(p=0,53)$ e IMC para la edad $(p=0,564)$, excepto en la talla para la edad (prueba de $\chi^{2}$ de independencia, $p=0,025$ ).

\section{Incidencias de infección respiratoria aguda y de enfermedad diarreica aguda}

Se evidenció que el número de niños con, al menos, un episodio de infección respiratoria aguda según el grupo de estudio fue de nueve menores $(9,677$ \%) en el grupo que tomó zinc aminoquelado, de 12 niños $(10,714 \%)$ en el grupo de sulfato de zinc y de $20(20,833 \%)$ en el que ingirió placebo.

Se encontró que los niños que tomaron leche con zinc aminoquelado presentaron las menores tasas y proporciones de incidencia tanto de infección respiratoria aguda como de enfermedad diarreica aguda (cuadro 4).

Se encontró una tasa de incidencia de infección respiratoria aguda menor en el grupo que recibió zinc aminoquelado (1,42 por 1.000 días-niño) 
Cuadro 4. Incidencia de infección respiratoria aguda y enfermedad diarreica aguda por grupo de estudio

\begin{tabular}{|c|c|c|c|c|c|c|c|c|c|c|c|c|}
\hline & \multicolumn{4}{|c|}{ Zinc aminoquelado (93 niños ) } & \multicolumn{4}{|c|}{ Sulfato de zinc (112 niños ) } & \multicolumn{4}{|c|}{ Placebo (96 niños ) } \\
\hline & $\mathbf{n}$ & $\%^{1}$ & $\begin{array}{c}\text { Persona- } \\
\text { tiempo de } \\
\text { observación }\end{array}$ & $\begin{array}{c}\text { Densidad de } \\
\text { incidencia² }^{2}\end{array}$ & $\mathbf{n}$ & $\% 1$ & $\begin{array}{c}\text { Persona- } \\
\text { tiempo de } \\
\text { observación }\end{array}$ & $\begin{array}{c}\text { Densidad de } \\
\text { incidencia }^{2}\end{array}$ & $\mathbf{n}$ & $\% 1$ & $\begin{array}{c}\text { Persona- } \\
\text { tiempo de } \\
\text { observación }\end{array}$ & $\begin{array}{l}\text { Densidad de } \\
\text { incidencia }^{2}\end{array}$ \\
\hline $\begin{array}{l}\text { IRA } \\
\text { EDA }\end{array}$ & $\begin{array}{l}9 \\
1\end{array}$ & $\begin{array}{l}9,6 \\
1,0\end{array}$ & $\begin{array}{l}6.305 \\
6.305\end{array}$ & $\begin{array}{l}1,42 \\
0,15\end{array}$ & $\begin{array}{r}12 \\
6\end{array}$ & $\begin{array}{r}10,7 \\
5,3\end{array}$ & $\begin{array}{l}7.629 \\
7.629\end{array}$ & $\begin{array}{l}1,57 \\
0,78\end{array}$ & $\begin{array}{r}20 \\
3\end{array}$ & $\begin{array}{r}20,8 \\
3,1\end{array}$ & $\begin{array}{l}6.061 \\
6.061\end{array}$ & $\begin{array}{l}3,30 \\
0,49\end{array}$ \\
\hline
\end{tabular}

IRA: infección respiratoria aguda; EDA: enfermedad diarreica aguda

n: número de episodios nuevos

1 Incidencia acumulada

${ }^{2}$ Tasa de incidencia por 1.000 niños

comparado con el grupo que recibió sulfato de zinc ( 1,57 por 1.000 días-niño); no se encontraron diferencias significativas entre las incidencias $\left(R R=0,90, \quad I C_{95 \%} \quad 0,382\right.$ a 2,153, $\left.p=0,999\right)$, el número necesario para tratar (6.666), y el porcentaje de reducción del riesgo relativo (10\%).

Se encontró que el número de niños que presentaron al menos un episodio de enfermedad diarreica aguda según el grupo de intervención fue de un menor $(1,075 \%)$ en el grupo que tomó zinc aminoquelado, de seis niños $(5,357 \%)$ en el grupo de sulfato de zinc y de tres infantes $(3,125 \%)$ en el que ingirió placebo.

En la enfermedad diarreica aguda, la tasa de incidencia fue menor en el grupo que recibió zinc aminoquelado (0,15 por 1.000 días-niño) que en el grupo que recibió sulfato de zinc $(0,78$ por 1.000 días-niño); no se encontraron diferencias significativas entre las incidencias $\left(\mathrm{RR}=0,20, \mathrm{IC}_{95 \%}\right.$ : $0,0043$ a 1,662, $p=0,361)$, el número necesario para tratar (1.666), y el porcentaje de reducción del riesgo relativo $(80 \%)$.

La tasa de incidencia de infección respiratoria fue menor en el grupo de zinc aminoquelado $(1,42$ por 1.000 días-niño) que en el grupo placebo $(3,3$ por 1.000 días-niño), encontrándose diferencias significativas entre las incidencias ( $R R=0,43, I_{95 \%}$ : $0,196$ a $0,950, p=0,049)$, el número necesario de tratar (531), y el porcentaje de reducción del riesgo relativo (57\%).

Se encontró una tasa de incidencia de enfermedad diarreica aguda menor en el grupo de zinc aminoquelado $(0,15$ por 1.000 .días-niño) que en el grupo placebo $(0,49$ por 1.000 días-niño); no se encontraron diferencias significativas entre las incidencias ( $R R=0,320, I_{95 \%} 0,006$ a 3,990, $p=0,346)$, la reducción del riesgo relativo (2.941), y el porcentaje de reducción del riesgo relativo (68\%).
Respecto a la tasa de incidencia de infección respiratoria aguda en el grupo de sulfato de zinc (1,57 por 1.000 días-niño), esta fue menor que la tasa de incidencia en el grupo placebo (3,3 por 1.000 días-niño); no se encontraron diferencias significativas entre las incidencias ( $R R=0,47, I C_{95 \%}: 0,233$ a 0,975 , $p=0,057)$, la reducción del riesgo relativo (578) y el porcentaje de reducción del riesgo relativo (53\%).

Al comparar la tasa de incidencia de enfermedad diarreica aguda en el grupo de sulfato de zinc (0,78 por 1.000 días-niño), esta fue mayor que la tasa de incidencia en el grupo placebo $(0,49$ por 1.000 niños-día); no se encontraron diferencias significativas entre las incidencias $\left(R R=1,58, I C_{95 \%}\right.$ : 0,339-9,819, $p=0,241$ ).

En la enfermedad diarreica aguda, la tasa de incidencia en quienes recibieron placebo $(0,49$ por 1.000 días-niño) fue menor comparada con la del grupo que recibió zinc (0,5 por 1.000 días-niño) (figura 1), sin diferencias estadísticas entre las incidencias ( $R R=1,01, I_{95 \%}: 0,231$ a $6,082, p=0,01$ ).

Se realizó el análisis de las incidencias de infección respiratoria aguda estratificando según si el niño sufría o no de alergia respiratoria y se encontró que sí se comportaba como posible variable confusora, con una diferencia de RR ajustado y sin ajustar de $13,3 \%$; por este motivo se calcularon nuevamente las incidencias y se halló que en los niños no alérgicos que consumieron zinc se presentaron menores tasas de incidencia de infección respiratoria aguda $(1,19$ por 1.000 días-niño) que en el grupo placebo (3,33 por 1.000 días-niño) con diferencias estadísticas ( $R R=0,35$, $\mathrm{IC}_{95 \%}$ : 0,177 a 0,725, p=0,005).

\section{Proporción de efectos secundarios en los grupos de estudio}

En total, 37 (12,29 \%) niños presentaron, por lo menos una vez, algún efecto secundario durante 
el periodo de observación. La prevalencia de efectos secundarios fue de $14,28 \%$ en el grupo de sulfato de zinc, de $13,54 \%$ en el grupo placebo y de $6,45 \%$ en el grupo de zinc aminoquelado. Al comparar los grupos de estudio, no se encontraron diferencias significativas (prueba de $\chi^{2}$ de independencia, $p=0,170$ ).

Al menos un episodio de vómito luego de tomar la leche se presentó en $10,7 \%$ de los niños del grupo que tomó sulfato de zinc, seguido por el grupo placebo $(10,4 \%) y$, en menor proporción, el grupo que recibió zinc aminoquelado $(5,4 \%)$. En ninguno de estos casos el vómito fue grave. No se encontraron diferencias significativas entre los grupos (prueba de $\chi^{2}$ de independencia, $p=0,246$ ) (figura 3).

Los niños que presentaron enfermedad diarreica presentaron el mayor porcentaje de vómito, comparados con los que no presentaron diarrea; sin embargo, al evaluar solo a los niños que no presentaron diarrea en los tres grupos, el grupo que tomó sulfato de zinc siguió siendo el que presentó vómitos con mayor frecuencia $(9,7 \%)$.

Al menos un episodio de dolor abdominal después de haber consumido la leche, se presentó en $3,6 \%$ de los niños en el grupo que tomó sulfato de zinc, seguido del grupo placebo $(3,1 \%)$ $\mathrm{y}$, en menor proporción, el grupo que recibió zinc aminoquelado $(1,1 \%)$. No se encontraron diferencias significativas entre los grupos (prueba de $\chi^{2}$ de independencia, $p=0,511$ ).

\section{Discusión}

El zinc juega un papel trascendente en el mantenimiento de la integridad celular y de la inmunidad, con una acción fundamental en el

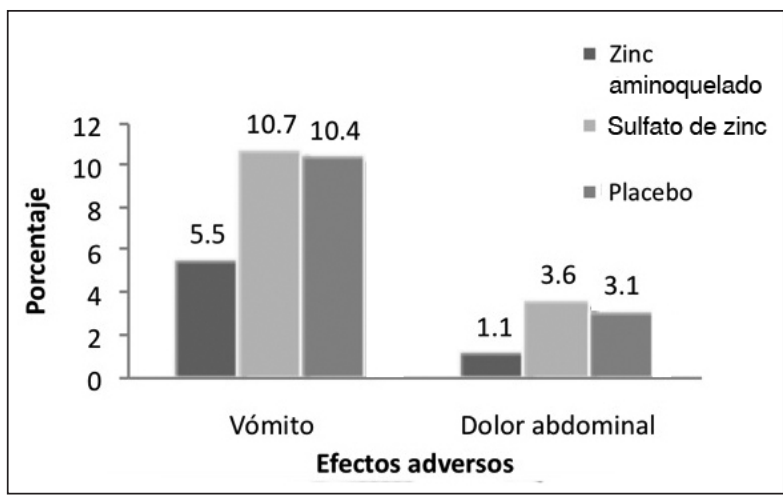

Figura 3. Proporción de efectos secundarios según grupo de estudio control y prevención de las infecciones $(1,12)$, ya que es un potente mediador en la resistencia del huésped (20-22). En el ámbito social, la deficiencia de zinc en países de bajos ingresos va ligada a situaciones como la pobreza, el hacinamiento y la ausencia de servicios públicos que elevan la vulnerabilidad de los niños; esto sumado a esquemas alimentarios inadecuados que generan deficiencia de minerales como el zinc, predispone a un mayor riesgo y gravedad de una variedad de enfermedades infecciosas $(1,2,23)$.

Se encontró que los niños que recibieron leche complementada con zinc (sulfato y aminoquelado) presentaron un riesgo de infección respiratoria aguda 2,22 menor que el de los niños que consumieron placebo, así como una reducción con el consumo de zinc de $55 \%$ en el riesgo de infección respiratoria aguda en comparación con los del grupo placebo; algo muy similar se encontró en los niños que tomaron leche complementada con zinc aminoquelado, el cual redujo en 57 $\%$ el riesgo de infección respiratoria aguda en comparación con el grupo placebo, así como una reducción del riesgo de infección respiratoria aguda de 2,32 comparada con la de los niños que no tomaron ningún tipo de zinc.

Similares efectos benéficos encontraron Vakili, et al., en niños que recibieron $10 \mathrm{mg}$ de sulfato de zinc, seis días por semana por cinco meses, pues hubo menor ocurrencia de resfriados en el grupo que tomó zinc $(p<0,001)$ (24). En otro estudio, Kurugol, et al., evaluaron la eficacia del sulfato de zinc (15 mg de sulfato de zinc, seis días por semana durante siete meses) en la reducción de la ocurrencia de resfriado en niños. La proporción de la incidencia de resfriado fue de $33 \%$ en el grupo placebo y de $14 \%$ en el grupo que consumió zinc (RR=0,4; $\left.I C_{95 \%}: 0,2-0,6, p \leq 0,05\right)$ (16). En el presente estudio se encontró menor incidencia no significativa en el grupo que consumió sulfato de zinc comparado con el grupo placebo $(R R=0,47$, $\mathrm{IC}_{95 \%}: 0,233$ a 0,975, p=0,057).

Por otro lado, en dos metanálisis, el de Singh, et al. (13), y el de Aggarwal, et al. (23), se encontró que el consumo de suplementos de zinc reducía la incidencia de infección respiratoria en forma significativa comparado con el placebo. Contrastada con la de nuestro estudio, la incidencia de infección respiratoria aguda también fue menor en los niños que consumieron algún tipo de zinc y se evidenció un riesgo menor de desarrollar dicha enfermedad $\left(R R=0,45, I C_{95 \%}: 0,247\right.$ a $\left.0,842, p=0,016\right)$. 
Además de los beneficios del zinc frente al resfriado común, varios ensayos clínicos han demostrado su utilidad en el manejo adjunto de la neumonía en niños menores de cinco años tal como lo describen Black (25), Brooks (26) y Bhandari (27).

No hay comprensión aún en torno a los mecanismos exactos por los que el zinc puede mejorar las infecciones de las vías respiratorias; sus efectos pueden deberse a la modulación del sistema inmunológico, así como por su acción sobre las membranas celulares, $y$ al hecho de ser un inductor en la producción de interferón y ayudar a modular las citocinas inflamatorias, las que a su vez pueden tener efectos beneficiosos sobre los síntomas de la infección respiratoria (22).

Con respecto a los criterios para evaluar la infección respiratoria aguda, al igual que en el presente estudio, varios ensayos clínicos previos han utilizado criterios basados en los signos y síntomas referidos por el niño o el cuidador sin hacer examen de la frecuencia respiratoria ni auscultación por parte de personal médico $(8,28)$.

En el análisis de las incidencias de infección respiratoria se observó que la variable alergia respiratoria se comportó como confusora; sin embargo, al comparar las incidencias en los niños que no tenían alergia respiratoria con las de los que sí la presentaban, siguió registrándose una diferencia significativa con menores tasas de incidencia en los niños que consumieron zinc comparados con los niños que no lo recibieron.

Con respecto a la enfermedad diarreica aguda, en este estudio se encontró que los niños que recibieron suplemento de zinc aminoquelado presentaron una reducción de 3,12 en el riesgo de enfermedad diarreica aguda y una de $68 \%$ en el riesgo de presentarla, comparados con los niños que no consumieron ningún tipo de zinc contrario a lo observado en el grupo que recibió sulfato de zinc, que mostró un riesgo de presentar enfermedad diarreica aguda 1,58 veces más que los niños que recibieron placebo. Esto es similar a lo encontrado con el zinc aminoquelado y difiere en lo concerniente a los resultados del sulfato de zinc. Black (25), Brown (8), Patel (11) y Bhutta (29) reportaron en sus metanálisis cómo la ingestión de zinc disminuía las tasas de incidencia de enfermedad diarreica aguda en los niños.

Con respecto a la enfermedad diarreica aguda, se sabe que, además de regular la función de los linfocitos $T$ y favorecer la recuperación inmunológica, el zinc tiene un efecto directo sobre la recuperación de las vellosidades intestinales, la actividad de las disacaridasas del borde en cepillo y el transporte intestinal de agua y electrolitos al actuar en los canales de potasio y cloro dependientes del cAMP (8).

La OMS sugiere $20 \mathrm{mg}$ de zinc al día por vía oral (en niños mayores de 12 meses) y $10 \mathrm{mg}$ al día (en niños menores a seis meses) durante10 a 14 días (9) como coadyuvante en el tratamiento de la enfermedad diarreica aguda (nivel de evidencia, IA) (30), ya que, además, disminuye la incidencia de diarrea en los dos a tres meses siguientes al episodio (9). Asimismo, se ha demostrado que el consumo de zinc reduce de manera significativa el número total de evacuaciones, la proporción de niños con evacuaciones líquidas o semilíquidas y la duración de la diarrea (nivel de evidencia II, B) (30).

Los resultados del presente estudio pueden responder a una menor absorción intestinal del sulfato de zinc al ser administrado con leche, dada la interacción entre el zinc y el calcio descrita en algunos estudios $(4,31)$, situación que pudo no haber ocurrido con el zinc aminoquelado debido a su estructura, la cual favoreció su absorción al no ser interferida por el calcio de la leche ni por otros micronutrientes del producto lácteo $(17,18,32,33)$.

Algunos estudios han comparado la absorción de otros compuestos de zinc en adultos sanos: Ashmead, et al., encontraron que la absorción intestinal del zinc aminoquelado era significativamente mayor en comparación con la absorción de sulfato de zinc, el óxido de zinc y el carbonato de zinc (32). Gandia, et al., encontraron que el zinc aminoquelado incrementaba significativamente la absorción oral del zinc $(43,4 \%)$ comparado con el gluconato de zinc (34). Schölmerich. et al., encontraron que la ingestión de zinc quelado con histidina aumentaba en una proporción de 1:2 a 1:12 la concentración sérica de zinc en comparación con la ingestión de sulfato de zinc (17).

Es de anotar que fue posible controlar $80 \%$ de las necesidades nutricionales diarias suministradas en los centros infantiles, ya que esta es una de las prioridades de la Fundación FAN; además, la minuta suministrada en todos los centros infantiles fue igual, lo cual garantizó un consumo similar de alimentos ricos en zinc y en otros nutrientes. Sin embargo, la alimentación que recibieron en sus casas los días sábado y domingo no se pudo controlar. 
En la evaluación del estado nutricional según los indicadores de medidas antropométricas y su respectiva puntuación $z$, se encontró que al comparar con los datos de la última Encuesta Nacional de Nutrición y Salud de Colombia (ENDS 2010), los niños del estudio presentaban porcentajes menores de talla baja para la edad, bajo peso para la edad y bajo peso para la talla, a excepción del porcentaje de niños obesos, el cual fue mayor en este estudio $(8,6 \%)$ en comparación con el reportado a nivel nacional $(5,2$ $\%)$. Esto posiblemente se debe a que los niños de recursos más bajos tienen una dieta altamente calórica y con pocas proteínas y vegetales, pero dado que la minuta diaria del estudio estuvo bien establecida, se registraron menos niños con desnutrición y un mayor porcentaje de menores obesos en comparación con las estadísticas nacionales (5).

En el análisis se encontró que los tres grupos de estudio presentaron aumento significativo en los valores de peso y talla al compararlos en cada grupo antes y después de la intervención. No se encontraron diferencias significativas en las medidas antropométricas según la puntuación $z$ entre los grupos al final del estudio. Algunos estudios previos han demostrado un efecto positivo sobre el aumento lineal en el peso y la talla, mas no sobre los índices de peso para la talla (4). Asimismo, el efecto sobre el crecimiento ha sido más evidente en niños desnutridos con talla para la edad y peso para la talla menor de 2 desviaciones estándar (4). Los estudios realizados en niños sin desnutrición no han encontrado cambios significativos en el crecimiento. Otros estudios han demostrado el efecto del zinc sobre la talla y el peso al suministrarlo por 12 meses 0 más $(25,35)$.

Debido al tiempo de seguimiento de solo 16 semanas establecido para este estudio y la posible intervención de otras variables que no se tuvieron en cuenta por no ser su objetivo, no hay suficiente información para decir que el aumento en el peso y la talla se debió al consumo de las leches fortificadas con zinc.

Con respecto a la aceptación de los compuestos de zinc administrados por vía oral, estos se consideran seguros cuando se consumen en dosis fisiológicas, a pesar de que efectos secundarios como el sabor metálico, las náuseas y el vómito hayan sido descritos en la literatura $(13,15,36,37)$, aunque con gran heterogeneidad entre los estudios en cuanto al tipo de compuesto de zinc suministrado, el tiempo de intervención y las dosis (37); tales efectos, sin embargo, no se han reportado como graves $(20,24,38)$. Esto se corrobora en el presente estudio, en el cual ningún niño presentó efectos secundarios que requirieran tratamiento adjunto o la suspensión del consumo del complemento con zinc suministrado.

Los presentes resultados no encontraron diferencias significativas con respecto al número de efectos adversos entre los grupos, posiblemente debido a que los compuestos de zinc se administraron en dos tomas diarias y en un vehículo lácteo que posiblemente contribuyó a enmascarar su sabor, lo cual los hizo más tolerables. Estos hallazgos son congruentes con los reportes en la literatura que no han encontrado diferencia al comparar los efectos secundarios del zinc y el placebo $(16,35,39)$; es de anotar que en estos estudios el compuesto de zinc utilizado fue el sulfato de zinc.

Llama la atención la mayor proporción de episodios de vómito y dolor abdominal en el grupo que tomó sulfato de zinc, situación que podría explicarse por una mayor intolerancia a este compuesto. Dicha diferencia en la tolerancia digestiva puede deberse, como lo plantean Singh, et al. (13), más que al zinc como tal, al tipo de vehículo utilizado; en el caso del presente estudio hubo más tolerancia al sulfato que al aminoquelado.

Los resultados del presente estudio no se pueden generalizar a toda la población infantil de Medellín sino solo a los niños entre dos y cinco años de los seis centros infantiles evaluados.

El examen físico es el método ideal para el diagnóstico específico de infección respiratoria aguda y, en el caso de la neumonía, la radiografía de tórax. Se requeriría mayor presupuesto que permitiera tener personal médico entrenado para examinar al menos semanalmente a los niños que presentaran síntomas y así poder definir el diagnóstico específico para el tipo de infección respiratoria.

Debido a las limitaciones presupuestales no fue posible obtener los niveles de zinc en sangre antes y después de la intervención, resultado que nos habría mostrado el mayor o menor incremento de los niveles en plasma de los dos compuestos de zinc evaluados.

\section{Agradecimientos}

A la Fundación de Atención a la Niñez (FAN), en especial a su nutricionista, Viviana Ramírez, a las 
directoras y profesoras de los centros infantiles y a todos los niños que participaron en la investigación.

\section{Conflicto de intereses}

Puede haber algún tipo de conflicto de intereses, ya que uno de los investigadores es empleado de una de las entidades financiadoras del estudio.

\section{Financiación}

Esta investigación fue financiada por la Universidad CES y Nutreo, S.A.S., Rionegro, Antioquia.

\section{Referencias}

1. Hess S, Lönnerdal B, Hotz C. Recent advances in knowledge of zinc nutrition and human health. Food Nutr Bull. 2009;30(Suppl.1):s5-9.

2. Gibson RS, Hess SY, Hotz C, Brown KH. Indicators of zinc status at the population level: a review of the evidence. $\mathrm{Br}$ J Nutr. 2008;99 (Suppl.3):S14-23. http://dx.doi.org/10.1017/ S0007114508006818

3. López de Romaña D, Castillo C, Diazgranados D. El zinc en la salud humana - II. Rev Chil Nutr. 2010;37:244-7. http:// dx.doi.org/10.4067/S0717-75182010000200014

4. Brown KH, Rivera JA, Bhutta Z, Gibson RS, King JC, Lönnerdal B, et al. International Zinc Nutrition Consultative Group (IZiNCG) technical document \#1. Assessment of the risk of zinc deficiency in populations and options for its control. Food Nutr Bull. 2004;25(Suppl.2):S99-203.

5. Fonseca Z, Heredia A, Ocampo R, Forero Y, Samiento O, Alvarez M, et al. Encuesta nacional de la situación nutricional en Colombia 2010 ENSIN. Bogotá: Davinci Editores; 2011.

6. Black RE, Cousens S, Johnson HL, Lawn JE, Rudan I, Bassani DG, et al. Global, regional, and national causes of child mortality in 2008: A systematic analysis. Lancet. 2010;375:1969-87. http://dx.doi.org/10.1016/S01406736(10)60549-1

7. DSSA. Estadisticas de morbilidad en Antioquia. Antioquia la más educada. Fecha de consulta: 22 de agosto de 2012. Disponible en: http://www.dssa.gov.co/index.php/ estadisticas/morbilidad.

8. Brown KH, Peerson JM, Baker SK, Hess SY. Preventive zinc supplementation among infants, preschoolers, and older prepubertal children. Food Nutr Bull. 2009;30(Suppl.1):S1240. http://dx.doi.org/10.1186/1471-2458-11-S3-S23

9. Robberstad B, Strand TA, Black R, Sommerfelt H. Cost effectiveness of zinc as an adjunct therapy for acute childhood diarrhoea in developing countries. Bull World Health Organ. 2004;82:523-31.

10. Brown KH, Hess SY. Impact of zinc fortification on zinc nutrition. Food Nutr Bull. 2009;30(Suppl.1):S79-107.

11. Patel AB, Mamtani M, Badhoniya N, Kulkarni H. What zinc supplementation does and does not achieve in diarrhea prevention: A systematic review and meta-analysis. BMC Infect Dis. 2011;11:122. http://dx.doi.org/10.1186/14712334-11-122

12. Yakoob MY, Theodoratou E, Jabeen A, Imdad A, Eisele TP, Ferguson J, et al. Preventive zinc supplementation in developing countries: Impact on mortality and morbidity due to diarrhea, pneumonia and malaria. BMC Public Health. 2011;11(Suppl.3):S23. http://dx.doi.org/10.1186/1471-245811-S3-S23

13. Singh M, Das RR. Zinc for the common cold. Cochrane Database Syst Rev. 2011;2:CD001364. http://dx.doi.org/10. 1002/14651858.CD001364.pub3

14. Lassi ZS, Haider BA, Bhutta ZA. Zinc supplementation for the prevention of pneumonia in children aged 2 months to 59 months. Cochrane Database Syst Rev. 2010;12:CD005978. http://dx.doi.org/10.1002/14651858.CD005978.pub2

15. Patro B, Golicki D, Szajewska H. Meta-analysis: Zinc supplementation for acute gastroenteritis in children. Aliment Pharmacol Ther. 2008;28:713-23. http://dx.doi.org/10.1111/ j.1365-2036.2008.03787.x

16. Kurugöl Z, Akilli M, Bayram N, Koturoglu G. The prophylactic and therapeutic effectiveness of zinc sulphate on common cold in children. Acta Paediatr. 2006;95:117581. http://dx.doi.org/10.1080/08035250600603024

17. Schölmerich J, Freudemann A, Köttgen E, Wietholtz H, Steiert B, Löhle E, et al. Bioavailability of zinc from zinchistidine complexes. I. Comparison with zinc sulfate in healthy men. Am J Clin Nutr. 1987;45:1480-6.

18. Aguilar F, Autrup H, Barlow S, Castle L, Crebelli R. Opinion on certain bisglycinates as sources of copper, zinc, calcium, magnesium and glycinate nicotinate as source of chromium in foods intended for the general population and foods for particular nutritional uses. EFSA Journal. 2008;718:3-26.

19. Ministerio de Salud y Protección Social. Resolución 2121 del 2010. Por la cual se adoptan los patrones de crecimiento publicados por la Organización Mundial de la Salud (OMS) en el 2006 y 2007 para los niños, niñas y adolescentes de 0 y 18 años de edad. 2010. Fecha de consulta: 23 de agosto de 2012. Disponible en: http://www.colombianutrinet.org/ index.php?option $=$ com_content\&view $=$ article $\& i d=630: r$ esolucion-2121-de-2010-adopcion-de-los-patrones-decrecimiento-de-la-oms\&catid=118\&ltemid=259.

20. Cuevas LE, Koyanagi A. Zinc and infection: A review. Ann Trop Paediatr. 2005;25:149-60. http://dx.doi.org/10. 1179/146532805X58076

21. Stefanidou M, Maravelias C, Dona A, Spiliopoulou C. Zinc: A multipurpose trace element. Arch Toxicol. 2005;80:1-9. http://dx.doi.org/10.1007/s00204-012-0843-1

22. Shankar AH, Prasad AS. Zinc and immune function: The biological basis of altered resistance to infection. Am J Clin Nutr. 1998;68(Suppl.2):447S-63.

23. Aggarwal R, Sentz J, Miller MA. Role of zinc administration in prevention of childhood diarrhea and respiratory illnesses: A meta-analysis. Pediatrics. 2007;119:1120-30. http://dx.doi. org/10.1542/peds.2006-3481

24. Vakili R, Vahendian M, Khoedaei G, Mahmoudi M. Effects of zinc supplementation in occurrence and duration of common cold in school aged children during cold season: A double-blind placebo-controlled trial. Iran J Pediatr. 2009;19:376-80.

25. Black RE. Zinc deficiency, infectious disease and mortality in the developing world. J Nutr. 2003;133(Suppl.1):1485S-9S.

26. Brooks WA, Santosham M, Naheed A, Goswami D, Wahed MA, Diener-West M, et al. Effect of weekly zinc 
supplements on incidence of pneumonia and diarrhoea in children younger than 2 years in an urban, low-income population in Bangladesh: Randomised controlled trial. Lancet. 2005;366:999-1004. http://dx.doi.org/10.1016/ S0140-6736(05)67109-7

27. Bhandari N, BahI R, Taneja S, Strand T, Mølbak K, Ulvik RJ, et al. Effect of routine zinc supplementation on pneumonia in children aged 6 months to 3 years: Randomised controlled trial in an urban slum. BMJ. 2002;324:1358. http://dx.doi. org $/ 10.1136 / \mathrm{bmj} .324 .7350 .1358$

28. Roth DE, Richard SA, Black RE. Zinc supplementation for the prevention of acute lower respiratory infection in children in developing countries: Meta-analysis and meta-regression of randomized trials. Int J Epidemiol. 2010;39:795-808. http://dx.doi.org/10.1093/ije/dyp391

29. Bhutta ZA, Black RE, Brown KH, Gardner JM, Gore S, Hidayat A, et al. Prevention of diarrhea and pneumonia by zinc supplementation in children in developing countries: Pooled analysis of randomized controlled trials. J Pediatr. 1999;135:689-97.

30. Ruiz V. Beneficios de la suplementación con cinc en el tratamiento de la diarrea en menores de cinco años de edad. Ciudad de La Habana: Instituto de Nutrición e Higiene de Los Alimentos; 2012.

31. Lönnerdal B. Dietary factors influencing zinc absorption. J Nutr. 2000;130(Suppl.5S):1378S-83S.

32. Ashmead H, Graff D, Ashmead H. Intestinal absorption of metal ions and chelates. Springfield, IL: Thomas; 1985. p. 118-25.
33. Muñoz EC, Rosado JL, López P, Furr HC, Allen LH. Iron and zinc supplementation improves indicators of vitamin A status of Mexican preschoolers. Am J Clin Nutr. 2000;71:789-94.

34. Gandia P, Bour D, Maurette J-M, Donazzolo Y, Duchène $\mathbf{P}$, Béjot $\mathbf{M}$, et al. A bioavailability study comparing two oral formulations containing zinc ( $\mathrm{Zn}$ bis-glycinate vs. $\mathrm{Zn}$ gluconate) after a single administration to twelve healthy female volunteers. Int J Vitam Nutr Res. 2007;77:243-8.

35. Lind T, Lönnerdal B, Stenlund H, Gamayanti IL, Ismail $D$, Seswandhana $\mathbf{R}$, et al. A community-based randomized controlled trial of iron and zinc supplementation in Indonesian infants: Effects on growth and development. Am J Clin Nutr. 2004;80:729-36

36. Allen L. Zinc and micronutrient supplements for children. Am J Clin Nutr. 1998;:495S-8S.

37. Lazzerini M, Ronfani L. Oral zinc for treating diarrhoea in children. Cochrane Database Syst Rev. 2013;1:CD005436. http://dx.doi.org/10.1002/14651858.CD005436.pub4

38. Bhandari N, Bahl R, Taneja S, Strand T, Molbak K, Ulvik RJ, et al. Substantial reduction in severe diarrheal morbidity by daily zinc supplementation in young North Indian Children. Pediatrics. 2002;109:e86. http://dx.doi. org/10.1542/peds.109.6.e86

39. Gupta DN, Mondal SK, Ghosh S, Rajendran K, Sur D, Manna B. Impact of zinc supplementation on diarrhoeal morbidity in rural children of West Bengal, India. Acta Paediatr. 2003;92:531-6.http://dx.doi.org/10.1111/j.16512227.2003.tb02501.x 